\title{
Mortality in interstitial lung disease: do race and skin colour matter?
}

\author{
Julie Morisset ${ }^{1}$, Yolanda Mageto ${ }^{2}$ and Ganesh Raghu ${ }^{3}$
}

Affiliations: ${ }^{1}$ Département de Médecine, Centre Hospitalier de l'Université de Montréal, Montréal, QC, Canada. ${ }^{2}$ Baylor University Medical Center, Center for Advanced Heart and Lung Disease, Dallas, TX, USA. ${ }^{3}$ Center for Interstitial Lung Disease, University of Washington, Seattle, WA, USA.

Correspondence: Julie Morisset, Centre Hospitalier de l'Université de Montréal, 1000 Saint-Denis, Montréal, Québec, H2X 0C1, Canada. E-mail: julie.morissetQumontreal.ca

@ERSpublications

African-Americans with interstitial lung disease exhibit a unique clinical phenotype http://ow.ly/NId830k4c0c

Cite this article as: Morisset J, Mageto Y, Raghu G. Mortality in interstitial lung disease: do race and skin colour matter? Eur Respir J 2018; 51: 1800892 [https://doi.org/10.1183/13993003.00892-2018].

In recent years, research surrounding health disparities has begun to focus more on the complex relationship between race, genetics and disease. Race has come to be recognised as an important factor, not only when discussing disparities and determining policy, but also in the diagnosis, approach and response to treatment.

Racial differences in diseases are important determinants of population health and may, unfortunately, lead to racial disparities [1]. Studying disease course and outcomes in patients of diverse races and ethnicities provides crucial knowledge for clinicians, researchers and public health organisations. Evaluating the impact of race on specific diseases can provide a greater understanding of risk factors and disease behaviour, and allow optimal patient management and healthcare quality.

In understanding the spectrum of incidence, prevalence, clinical manifestations and outcome of people ("human race") worldwide confronted with disease, and related to "race", one must be reminded that the commonly used terms "race", "ethnicity" and "Caucasian" are distinct from one another and have different meaning and significance [2-4]. Considering the concept of race and ethnicity are consistently evolving, while racial and ethnic categories used in the medical literature are often imprecise and based on socially acceptable norms, these categories can under-represent the real heterogeneity of patient populations [5]. For example, when the term "Caucasian" is loosely used to describe patient race or ethnicity, which are not synonymous terms, one takes for granted that the patient is of European origin (descent) and thus is "white". The word "Caucasian" in fact refers to people originating from the Caucasoid regions and that not only includes those of European origin but also those from Afghanistan, Pakistan and the subcontinent of India [2]. When used in research, the commonly used term "Caucasian", relating to the people of European origin with white skin, is only a myth. The definitions of the terms and categories, regarding race, should be more clearly stated to ensure readers can identify the correct groups of patients and interpret correctly the conclusions [2].

Multiple studies have shown that African-American race is known to be associated with poorer outcomes in several different types of medical conditions when compared to Caucasians of European origin. Notably, African-Americans with cancer, cardiac sarcoidosis or cardiovascular disease experience poorer survival than Caucasians of European origin due to either lack of access, different response to approved therapies or biases in diagnosis and treatment from the medical community [6-8]. 
Quantifying the race effect represents a highly challenging task considering the "immutable characteristics" of race [9, 10]. Additionally, race shares a complex relationship with many different individual characteristics such as socio-economic status, diet, genetics, geographic location, religion, cultural habits and countless other variables [9]. When race is the exposure of interest, the classic epidemiological mission of accounting for all potential confounders becomes more complex as one navigates all the all the intricate ramifications and associations of race.

Despite the advances in the field of interstitial lung disease (ILD), several questions regarding disease pathogenesis and epidemiology remain unanswered. To date, most of the large epidemiological studies and clinical trials in ILD have been conducted in predominantly white patient populations of European descent [11, 12], potentially limiting the generalisability of findings to patients from other parts of Caucasoid regions or of other races. For example, utilising the 2011 criteria for diagnosis of idiopathic pulmonary fibrosis (IPF) [13], a recent prospective study in India documented that nearly $15 \%$ of patients of over 1000 patients of incident cases of ILD had IPF [14]. Longitudinal data from patients with IPF and other ILD of Indian origin living in India are needed to understand if the clinical outcomes and disease behaviour in this cohort of "Caucasians of Indian origin" are similar or different from the Caucasians of European origin.

In this issue of the European Respiratory Journal, AdEgunsoye et al. [15] use a cohort from five different centres in the USA to describe the characteristics, risk of hospitalisation and mortality in African-American patients with ILD. The main analysis of this novel study was to investigate the association between African-American race and mortality using a propensity score analysis to minimise bias by confounding. Each African-American patient was matched to patients of other races based on their probability of identification as African-American. Covariates to be included in the propensity score were cleverly selected based on their known association with race and mortality.

The results as reported by ADEGUNSOYe et al. [15] highlight how race does in fact matter in the disease course of patients with ILD. African-American patients exhibited several differences in their baseline characteristics and outcomes when compared to the population of non-African-Americans. Interestingly, African-Americans patients were younger, more likely to be female and less likely to have a diagnosis of IPF or chronic hypersensitivity pneumonitis. Moreover, African-American patients with all ILD had better overall survival than non-African-American patients. By contrast, patients with a diagnosis of IPF had similar survival in both groups. These mortality results remained consistent using several different sensitivity analyses (across different patient subgroups, covariate adjustment in various multivariable Cox Models with and without propensity score use, and accounting for missing data).

The findings uncovering distinct clinical features in African-Americans patients with ILD are intriguing and open several questions for discussion. Are there regional, geographical and cultural differences in ILD? Would African-Americans living in other parts of the country also exhibit this phenotype? What is the influence of other races in ILD? What are the genetic or pathophysiological mechanisms explaining the clinical differences between races? It is interesting to note that their data suggested that patients of Asian and Hispanic origins fared worse than those of Caucasian (white) or African Americans. While these were very small numbers it gives credence to the issue of looking at ILD not only in terms of diagnosis but also in terms of ethnicity and race. We are hopeful this study will pave the way for further research aiming to elucidate how race can influence disease and outcomes in ILD.

The lower occurrence of IPF in African-Americans found in this study confirms previous work that showed the majority of patients with IPF are Caucasians of European origin [16]. Clinicians should have a high level of suspicion for non-IPF diagnoses when they evaluate African-American patients with ILD. However, additional multicentre studies including greater sample size of African-American patients with IPF are needed to better characterise IPF characteristics and behaviours in this patient population.

Key considerations should be kept in mind when interpreting the results presented in the report by AdEGUNSOYe et al. [15]. First, despite the authors' best effort to deal with potential confounding in the relationship between race and survival, no statistical method is perfect when dealing with this delicate situation. Propensity scores are increasingly being used in observational studies and represent an attractive choice when multiple covariates need to be included as confounders [17, 18]. However, an extensive knowledge of the predictors of exposure, in this case African-American race, is required to adequately select the covariates to be included in the propensity score [19]. In this study, complete data on the socio-economic status of patients were not available for the authors to include in their propensity score. The exclusion of such variables and other unmeasured confounding from the propensity score may have influenced the results. Additionally, the possibility of misclassification of ILD (e.g. some African-Americans patients with sarcoidosis could have been wrongly attributed a diagnosis of hypersensitivity pneumonitis) may have contribute to the observed results of better outcome in 
African-American patients. Finally, the study included patients from one region in the USA that is highly populated by African-Americans. Nevertheless, it is a good starting point to plan additional research looking at race, not only in a single state or centre, but also to begin to take into consideration the underlying regional and ethnic differences. Moreover, when dealing with data involving race we have to recognise the issue of multi-ethnic individuals. African-Americans in the USA have evolved into a multi-ethnic group subsequent to the historical days of the entry of the Africans into the USA. Genetic studies will also be very enlightening and may demonstrate regional differences. Therefore, one must be careful with the generalisation of any of these studies.

In conclusion, AdEgunsoye et al. [15] have described how African-Americans with ILD exhibit a unique clinical phenotype and raised several further challenging and exciting questions about the rationale behind those differences. Their report surfaces important considerations in understanding the incidence, prevalence, clinical manifestation and outcomes of patients with ILD of different race, colour and ethnicity, besides cultural, socioeconomic and regional variabilities. It is hoped that this work will provoke studies aiming to better understand the role of race and ethnicity along with the intrinsic and extrinsic factors in the individuals manifesting ILD. Further research exploring the mechanistic and pathobiological pathways responsible for the clinical difference between races and ultimately leading to a more comprehensive and personalised care of patients with ILD is warranted [20].

Conflict of interest: None declared.

\section{References}

1 Fiscella K, Franks P, Gold MR, et al. Inequality in quality: addressing socioeconomic, racial, and ethnic disparities in health care. JAMA 2000; 283: 2579-2584.

2 Bhopal R, Donaldson L. White, European, Western, Caucasian, or what? Inappropriate labeling in research on race, ethnicity, and health. Am J Public Health 1998; 88: 1303-1307.

Winker MA. Measuring race and ethnicity: why and how? JAMA 2004; 292: 1612-1614.

Kaplan JB, Bennett T. Use of race and ethnicity in biomedical publication. JAMA 2003; 289: 2709-2716.

Schwartz RS. Racial profiling in medical research. N Engl J Med 2001; 344: 1392-1393.

Zeng C, Wen W, Morgans AK, et al. Disparities by race, age, and sex in the improvement of survival for major cancers: results from the National Cancer Institute Surveillance, Epidemiology, and End Results (SEER) Program in the United States, 1990 to 2010. JAMA Oncol 2015; 1: 88-96.

7 Roger VL, Go AS, Lloyd-Jones DM, et al. Heart disease and stroke statistics - 2011 update: a report from the American Heart Association. Circulation 2011; 123: e18-e209.

8 Swigris JJ, Olson AL, Huie TJ, et al. Sarcoidosis-related mortality in the United States from 1988 to 2007. Am J Respir Crit Care Med 2011; 183: 1524-1530.

9 Sen M, Wasow O. Race as a bundle of sticks: designs that estimate effects of seemingly immutable characteristics. Annu Rev Polit Sci 2016; 19: 499-522.

10 VanderWeele TJ, Robinson WR. On the causal interpretation of race in regressions adjusting for confounding and mediating variables. Epidemiology 2014; 25: 473-484.

11 Richeldi L, du Bois RM, Raghu G, et al. Efficacy and safety of nintedanib in idiopathic pulmonary fibrosis. $N$ Engl J Med 2014; 370: 2071-2082.

12 King TE Jr, Bradford WZ, Castro-Bernardini S, et al. A phase 3 trial of pirfenidone in patients with idiopathic pulmonary fibrosis. N Engl J Med 2014; 370: 2083-2092.

13 Raghu G, Collard HR, Egan JJ, et al. An official ATS/ERS/JRS/ALAT statement: idiopathic pulmonary fibrosis: evidence-based guidelines for diagnosis and management. Am J Respir Crit Care Med 2011; 183: 788-824.

14 Singh S, Collins BF, Sharma BB, et al. Interstitial lung disease in India. Results of a prospective registry. Am J Respir Crit Care Med 2017; 195: 801-813.

15 Adegunsoye A, Oldham JM, Bellam SK, et al. African-American race and mortality in interstitial lung disease: a multicentre propensity-matched analysis. Eur Respir J 2018; 51: 1800255.

16 Swigris JJ, Olson AL, Huie TJ, et al. Ethnic and racial differences in the presence of idiopathic pulmonary fibrosis at death. Respir Med 2012; 106: 588-593.

17 Austin PC. An introduction to propensity score methods for reducing the effects of confounding in observational studies. Mult Behav Res 2011; 46: 399-424.

18 Joffe MM, Rosenbaum PR. Invited commentary: propensity scores. Am J Epidemiol 1999; 150: 327-333.

19 Brookhart MA, Schneeweiss S, Rothman KJ, et al. Variable selection for propensity score models. Am J Epidemiol 2006; 163: 1149-1156.

20 Brownell R, Kaminski N, Woodruff PG, et al. Precision medicine: the new frontier in idiopathic pulmonary fibrosis. Am J Respir Crit Care Med 2016; 193: 1213-1218. 